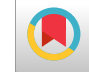

\title{
Prominent Prognostic Factors in Aggressive Breast Cancer: A Review
}

\author{
Kazem Nejati (iD) ${ }^{1,}$, Sedigheh Fekri Aval ${ }^{2,3}$, Mohammadreza Alivand ${ }^{4}$, Abolfazl Akbarzadeh ${ }^{5}$ and \\ AmirAhmad Arabzadeh (iD ${ }^{6, * *}$ \\ ${ }^{1}$ Pharmaceutical Sciences Research Center, Ardabil University of Medical Sciences, Ardabil, Iran \\ ${ }^{2}$ Biotechnology Research Center, Tabriz Branch, Islamic Azad University, Tabriz, Iran \\ ${ }^{3}$ Department of Biology, Tabriz Branch, Islamic Azad University, Tabriz, Iran \\ ${ }^{4}$ Department of Medical Genetics, Faculty of Medicine, Tabriz University of Medical Sciences, Tabriz, Iran \\ ${ }^{5}$ Department of Medical Nanotechnology, Faculty of Advanced Medical Sciences, Tabriz University of Medical Sciences, Tabriz, Iran \\ ${ }^{6}$ Department of Surgery, School of Medicine, Ardabil University of Medical Sciences, Ardabil, Iran \\ "Corresponding author: Pharmaceutical Sciences Research Center, Ardabil University of Medical Sciences, Ardabil, Iran. Email: k.nejati@arums.ac.ir \\ "Corresponding author: Department of Surgery, School of Medicine, Ardabil University of Medical Sciences, Ardabil, Iran. Email: amir_medico@yahoo.com \\ Received 2020 September 06; Revised 2021 August 07; Accepted 2021 August 09.
}

\begin{abstract}
Context: Breast cancer (BC) is the most common cancer in women worldwide. Hereditary susceptibility created by mutations in autosomal dominant genes is responsible for 5 to $10 \%$ of all BC cases in women. Recent studies have identified genes associated with increased risk for aggressive $\mathrm{BC}$, providing the basis for better risk management.

Evidence Acquisition: The latest information in National Center for Biotechnology Information (NCBI), Google Scholar, ScienceDirect, and Scopus were the main databases for finding articles. A combination of keywords of 'metastasis', 'invasion', 'aggressive breast cancer', 'prognostic factor', 'mutation', and 'cancer treatment' was searched in the databases to identify related articles. Titles and abstracts of the articles were studied to choose the right articles.

Results: Mutations in breast cancer type 1 susceptibility protein (BRCA1) and breast cancer type 2 susceptibility protein (BRCA2) genes are two central players related to the high risk of BC. Mutation in tumor protein p53 (TP53) is another important mutation that leads to triple-negative BC. Although the majority of BC types are not associated with high-throughput mutant genes such as BRCA1, BRCA2, and TP53, they are associated with low-throughput genes, including DNA repair protein Rad50 (RAD50), Nijmegen breakage syndrome gene (NBS1), checkpoint kinase 2 (CHEK2), BRCA1-interacting protein 1 (BRIP1), E-cadherin gene (CDH1) and PALB2, UCHL1, aldehydedehydrogenase1A3 (ALDH1A3), androgen receptor (AR), 5-bisphosphate 3-kinase (PIK3CA), phosphatidylinositol-4, and luminal gene expression that are generally mutated in the global population. High tumor mutational burden (TMB) was associated with improved progression-free survival.

Conclusions: The lymph node status, early tumor size, ER, PR, human epidermal growth factor receptor-2 (HER2), and Ki-67 are conventional prognostic factors for BC. However, these factors cannot exactly predict the aggressive behavior of BC. Hence, in this review, we discussed new prognostic factors of aggressive BCs that are useful for the treatment of patients with BC.
\end{abstract}

Keywords: Aggressive Breast Cancer, Prognostic Factors, Metastases, Mutations, Cancer Treatment

\section{Context}

Breast cancer $(\mathrm{BC})$ is a significant reason for cancerrelated death among women; approximately 40000 deaths occur per year in the United States (1). About 1 in 12 women in developed countries is diagnosed with BC during her life. Despite advancements in the primary diagnosis and therapy of BC such as smart drug delivery by nanoparticles (2-4), immunotherapy, and gene therapy, mortality is about $100 \%$ for that $20 \%$ of patients with metastases (5). Currently, early tumor size and the presence of lymph node metastasis (LNM) are the most significant prognostic markers of BC. However, the precision of these typical indicators that are used in the clinical setting is not high, thereby leading to the ineffective application of systemic treatment. BC has various presentations with extensive diverseness in its morphological features, clinical subsequences, prevalence tendency, and subtypes. Age is the most significant risk factor of BC, followed by a positive family history of BC. Hereditary susceptibility created by mutations in BRCA1/2 genes is responsible for 5 to $10 \%$ of all BCs among women (6). The genetic alterations in $\mathrm{BC}$ can be classified into two separate categories. The first category is gain-of-function mutations in proto-oncogenes. These mutations stimulate cell growth and division. The second category of genetic alterations is loss-of-function mutations in tumor suppressor genes that lead to the lack of ability to repair DNA 
after damage, uncontrollable cell growth, and lack of cell cycle checkpoints (7). Women inheriting loss-of-function mutations have an up to $85 \%$ risk of aggressive $\mathrm{BC}$ to the age of 70 years. Mutations in BRCA1/2 genes are two central players related to the high risk of BC. Germ-line mutations in these two genes are responsible for $16 \%$ of all hereditary $\mathrm{BC}$ cases. The breast cancer type 1 susceptibility protein (BRCA1) and 2 genes are tumor suppressor genes that are involved in DNA recombination and transcriptional regulation (8). Another significant mutation can occur in tumor protein p53 (TP53), leading to a triple negative BC (TNBC). TNBC is the most aggressive form of $\mathrm{BC}$, whose management is considered a medical challenge (9). TP53, phosphatase and tensin homologue (PTEN), Nijmegen breakage syndrome gene (NBS1), ataxia-telangiectasia mutated (ATM), and serum thymidine kinase 1 (STK1) are involved in hereditary BC syndromes like Li-Fraumeni (TP53), Cowden syndrome (PTEN), Nijmegan Breakage Syndrome (NBS1), Louis-Bar Syndrome (ATM), and Peutz Jeghers (STK1/LKB1). The majority of BC cases are not associated with high-throughput mutant genes such as BRCA1, breast cancer type 2 susceptibility protein (BRCA2), and TP53 (10). Low-throughput genes, including NBS1, checkpoint kinase 2 (CHEK2), DNA repair protein Rad50 (RAD50), E-cadherin gene (CDH1), partner and localizer of BRCA2 (PALB2), and BRCA1-interacting protein 1 (BRIP1) that are often mutated in the global population mostly contribute to BC extension (11). Recent studies have also shown that miRNAs can regulate signaling pathways negatively or positively, thereby affecting tumorigenesis and various aspects of cancer progression, particularly BC (12-14). Cancer-causing driver mutations confer selective clonal growth advantage and oncogenic potential to cells. The ability to invade and pass basement membranes of the endothelium and secondary organ sites is a hallmark of metastatic cancer cells. Distant metastasis or local invasion, rather than an early tumor, is responsible for the mortality of patients with cancer. Metastasis is a complex process, involving escape from an initial tumor, infiltration into lymph-vascular space, survival in the circulatory system, extravasation, and growth in the new site as a secondary tumor (15). The cellular mechanisms of metastasis are not well understood, but increased cell motility is one of the reasons. Increased motion of cancer cells has been related to a poor prognosis in human cancer and larger metastatic potential in animal models. However, newly reported research studies maintain the importance of targeted therapies on response to treatment in poor prognosis patients with BC. Barroso-Sousa et al. in 2020 in a cohort study demonstrated that high tumor mutational burden (TMB) and PTEN alterations correlate with response to anti-PD-1/L1 therapies among patients with metastatic triple-negative BC (mTNBC). This study showed that high TMB was associated with improved progression-free survival, while PTEN alterations were related to decreased responses and progression-free and survival among these patients (16). In other studies of pre-treatment and post-treatment biopsies from patients with triple-negative $\mathrm{BC}$ (TNBC) demonstrated androgen receptor (AR) expression, 5-bisphosphate 3-kinase (PIK3CA), phosphatidylinositol-4, and luminal gene expression of mutated genes in these pathologic conditions due to various responses in patients. For example, patients with luminal AR (LAR) subtype tumors possess clinical benefits and better prognosis and prolonged progression-free survival besides alteration in cell signaling-related gene expression (17). Considering the variability in clinical development of $\mathrm{BC}$, identification of new molecular markers of tumor behavior at the time of diagnosis is important. Few markers of $\mathrm{BC}$ development have been confirmed to be clinically beneficial (18). Progesterone receptor (PR) and estrogen receptor (ER) are extremely predictive in patients with $\mathrm{BC}$ that will profit from endocrine treatment; however, they are weak prognostic factors. ErbB2 [human epidermal growth factor receptor-2 (HER2)/neu] amplification or overexpression, Urokinase-type plasminogen activator receptor (UPAR), Ki-67, STAT3, and cathepsin D are other tumor markers that have been considered for prognosis in BC (19). Therefore, there is a need for new prognostic factors that are more precise and valid. In this review, we discuss new prognostic factors of aggressive BC.

\section{Evidence Acquisition}

The latest information in National Center for Biotechnology Information (NCBI), Google Scholar, ScienceDirect, and Scopus were the main databases for finding articles. A combination of keywords of 'metastasis', 'invasion', 'aggressive breast cancer', 'prognostic factor', 'mutation', and 'cancer treatment' was searched in the databases to identify related articles. Titles and abstracts of the articles were studied to choose the right articles. Finally, 92 articles were selected to study in detail and use as references.

\section{Results}

\section{1. $P 53$}

P53, a nuclear phosphoprotein, is encoded by the TP53 gene that is located at chromosome 17p13.1 (20). In 1979, p53 was identified as the first tumor suppressor gene. P53 can act as a negative regulator of cell growth and prevents neoplastic development by inhibiting abnormal cell proliferation. P53 has been identified as a transcription factor, which regulates numerous target genes involved in cell cycle arrest and induction of apoptosis $(21,22)$. Mutation in p53 results in loss of these functions. Alterations in the p53 gene are found in a variety of human tumors, including 
BC. Mutation in this gene is considered a primary event in the progression of $\mathrm{BC}$. The frequency of p53 mutations is about 20 to $30 \%$ in BC. Allelic imbalance on the short arm of chromosome 17 eliminates the tumor suppressor function of p53 in more than half of BCs (23). Mutant p53 protein gains novel tumor-promoting activities by transactivation of target genes related to apoptosis, cell proliferation, tissue invasiveness, and tumorigenesis.

Accordingly, since p53 mutations are prevalent in patients with $\mathrm{BC}$, it is thought which mutations of p53 could be related to more aggressive cancers or those with a high risk of distant metastasis and, therefore, might be a prognostic factor in forecasting future recurrence (24). The relationship between overexpression of p53 protein and disease-free survival has been investigated in several studies. In some types of carcinomas, UCHL1 forms a complex with p53/MDM2/ARF and induces apoptosis through aberrant methylation of the CpG islands in the ubiquitination process in tumor progression (25).

Among novel therapeutic target molecules, UCHL1 is considered a candidate oncoprotein for prolonging BC metastasis. UCHL1-enriched exosomes are significantly increased in sera of patients with triple-negative BC, the aggressive type of BC. Mechanistically, UCHL1 induces TGF$\beta$ signaling-induced metastasis and TGF- $\beta$ type 1 receptor and SMAD2 through ubiquitination and increases extravasation and migration in $\mathrm{BC}$ correlated with the paracrine role for UCHL1 in the progression of the tumor. These responses of cells are potentially suppressed by specific UCHL1 inhibitors and 6RK73. Furthermore, animal model experiments on zebrafish and BC xenograft models indicated the metastatic-inducing role for UCHL1 (26).

\subsection{Matrix Metalloproteinases (MMPs)}

Tumor cell local invasion into surrounding matrix and metastasis to distant organs require accurate coordination of adhesion to extracellular matrix (ECM) and controlled degradation of its protein components. Both processes are significant properties of malignant breast tumors. To invade, neoplastic cells require to infiltrate through the basement membrane and remove ECM borders. In this context, protease enzymes play a major role since they can hydrolyze protein components of ECM, thereby supporting cancer cell invasion (27). Among several proteinases that are involved in tissue lysis, members of the family of MMPs are important. MMP2 (Gelatinase A, $72 \mathrm{kDa}$ ), MMP9 (Gelatinase $\mathrm{B}, 92 \mathrm{kDa}$ ), and zinc-dependent endopeptidases of the MMP family play a critical role in the degradation of basement membrane collagen IV, the first crucial obstacle that is breached through cancer cells when they become invasive. MMP2 and MMP9 are released from cells as a zymogen and cleaved to the active form (28). Secretion of these two enzymes can be induced by a variety of factors, including growth factors, inflammatory cytokines, hormones, and oncogene products. The activity of MMP2 and MMP9 is controlled by interaction with tissue inhibitors of metalloproteinases (TIMPs), including tissue inhibitor of metalloproteinases 2 (TIMP2) and tissue inhibitor of metalloproteinases 1 (TIMP1), respectively. MMP2/9 is involved in the initiation of tumor growth and metastasis, particularly in BC. MMP2 is expressed in many primary stages of BC and is believed to play a key role in the first steps that result in tumor formation (27). MMP9 is also expressed in BC and appears to be related to the growth of the tumor and metastasis (29). MMPs in BC correlated with shortened recurrencefree survival, poor prognosis, and invasiveness of the disease. Also, these proteases are identified as prognostic factors in patients with aggressive $\mathrm{BC}(30)$.

\subsection{Enhancer of Zeste Homolog 2 (EZH2)}

$\mathrm{EZH} 2$, a polycomb group protein, is a methyltransferase and catalytic subunit of the Tissue inhibitor of metalloproteinases 2 (PRC2) enzyme complex. The PRC2 is involved in tumorigenesis and gene silencing by histone H3 lysine-27 methylation (31). Histone methylation by EZH2 results in chromatin changes, which mark DNA sequences for methylation through DNA methyltransferases. Also, core histone modifications lead to the formation of a constant, repressive chromatin structure, and silencing of developmental regulators, tumor suppressors, and differentiation-specific genes. EZH2 overexpression is associated with BC aggressiveness, poor prognosis, and decreased metastasis-free survival in patients with BC (32). Collett et al. found strong associations between overexpression of EZH2 and properties of aggressive BC, such as tumor cell proliferation, metastatic spread at diagnosis, locally advanced disease, and reduced patients' survival. These findings, thus, suggest the utility of EZH2 as a novel prognostic factor for BC (33).

\subsection{Fascin}

Manipulation of the actin cytoskeletal network results in increasing cell movement that is one of the most important cellular mechanisms, which control metastasis. Fascin, an actin cross-linking protein, is a member of the cytoskeletal protein family that localizes to microspikes, lamellipodia, and filopodia and has a key role in the adhesion and motility of cells. Fascin is expressed in normal specialized cells such as dendritic and neuronal cells during embryonic development, but its expression is weak in normal epithelial cells (34). However, overexpression of fascin has been detected in different types of human epithelial tumors such as breast carcinomas. Fascin promotes metastasis through facilitating membrane protrusions such as filopodia during the invasion of cancer cells and regulating metastasis-related genes (35), particularly upregulation of Nuclear factor kB (NF- $\kappa \mathrm{B})$ activity and downregulation of the breast cancer metastasis suppressor 1(BRMS1) as 
well as induction of UPA, MMP2, and MMP9 expression (36). There is evidence suggesting that growth factors and cytokines such as epidermal growth factor (EGF), interleukin 6 (IL-6), and transforming growth factor $\beta$ (TGF $\beta$ ) induce overexpression of fascin in cancer cells (35).

A study in patients with BC indicated that expression of fascin correlated inversely with PR and ER expression. These observations suggest a feasible association between augmented cell movement, hormone receptor negativity, and overexpression of fascin in aggressive BC (37).

Trials of $\mathrm{BC}$ have shown that the expression of fascin may be controlled by overexpression of HER2. Grothey et al. have shown that overexpression of HER2 in cancerous cells of the breast is related to graphic increases in mRNA and protein levels of fascin. The induction of fascin in response to HER2 may play a role in the induction of more aggressive and metastatic properties in BC cells. Generally, the expression of fascin in BC correlates with shorter disease-free survival and aggressive clinical behavior and can be considered a new prognostic factor for primary identification of aggressive carcinomas (38).

\subsection{Hypoxia-Inducible Factor 1(HIF1)}

Hypoxia, which is a loss of oxygen in tissues, is demonstrated in most solid tumors. Intratumoral hypoxia is created by cancer cells unlimited growth, lack of functional blood vessels, and deterioration of microcirculation. Hypoxia is associated with a potential for metastasis, poor prognosis, and enhanced malignancy in some types of carcinomas such as breast carcinomas. Hypoxic tumor microenvironments induce genetic and adaptive changes in tumor cells. These cells can adapt to the hypoxic conditions by the action of transcription factor HIF1 (39). HIF1 plays a major role in early tumor development and vascularization. HIF1 is a heterodimeric protein composed of HIF- $1 \alpha$ and HIF- $1 \beta$ subunits. Both of these subunits belong to the basic helix-loop-helix (bHLH) periodic acid Schiff superfamily. HIF- $1 \beta$ is constitutively expressed, whereas HIF- $1 \alpha$ is continuously degraded through the ubiquitinproteasome pathway under normoxia and its expression levels increase during hypoxia (40).

Under normoxia, HIF-1 $\alpha$ is hydroxylated through specific prolyl-hydroxylases. The hydroxylated form binds to von Hippel-Lindau protein, thus targeting HIF-1 $\alpha$ for degradation by the proteasome (41). Under hypoxia, HIF-1 $\alpha$ stabilization occurs by inhibition of hydroxylase activity and, consequently, its intracellular levels increase. Upon stabilization, HIF- $1 \alpha$ is translocated into the nucleus, where it heterodimerizes with HIF-1 $\beta$ to form a functional HIF1 transcription complex. This complex binds to hypoxiaresponsive elements (HREs) that are present in the target genes promoter region (42).

HIF-1 $\alpha$ controls the expression of genes associated with several critical biological processes, such as angio- genesis, cell growth, glycolytic metabolism, apoptosis, and invasion. Alterations in the expression patterns of these downstream genes counteract hypoxia, enhance oxygen delivery, and activate oxygen-independent alternative metabolic pathways (43).

In $\mathrm{BC}$, overexpression of HIF-1 $\alpha$ has been found in invasive carcinomas. Moreover, direct associations between HIF-1 $\alpha$ overexpression and mortality have been demonstrated in patients with aggressive BC. Several studies have revealed that increased expression of HIF- $1 \alpha$ is a new prognostic factor in aggressive forms of BC (44).

\subsection{Human Telomerase Reverse Transcriptase (hTERT)}

Telomerase is a specialized RNA-dependent DNA polymerase that is located in the nucleus. It is a ribonucleoprotein enzyme complex, which consists of two major subunits, including reverse transcriptase (hTERT), the catalytic subunit, and hTR, the internal RNA component. The hTR is expressed in both normal and cancer cells, whereas hTERT is highly expressed in cancerous cells but not in normal cells. The hTERT expression closely correlates with telomerase activity and serves as an indicator of telomerase activity. Telomerase synthesizes telomeric repeated sequence after the division of cell and maintains telomere length and genome stability, thereby resulting in cancer cells immortalization and cancer progression (45). The hTERT expression is regulated at the gene transcription level through several epigenetic modifications at promoter sites, including promoter methylation and histone acetylation. Without activation of telomerase, each round of cell division leads to shortening of the telomeres. Progressive shortening of telomeres induces telomere dysfunction and chromosome instability (46).

Telomerase is active in 80 to $90 \%$ of cancer types, including BC and its upregulation is detected as a primary event during development to malignancy of breast tumor. Ceja-Rangel et al. found that invasive BC cells have very short telomeres, and telomerase is highly activated in these cells (47). Poremba et al. reported that enhanced expression levels of telomerase core components are related to shorter overall survival (48). These findings suggest that hTERT is a promising emerging prognostic factor in aggressive BCs.

\subsection{Inhibitor of Differentiation/DNA Binding (Id)}

Inhibitor of differentiation/DNA binding (Id) HLH proteins are predominant negative regulators of bHLH transcription factors. BHLH transcription factors are essential regulators expressed by tissue-specific genes in some mammalian and non-mammalian organisms. These factors as homo- or heterodimers bind to DNA and regulate transcription of target genes that carry E-boxes in their promoters (49). Although Id proteins are dimerized with bHLH proteins, these heterodimers are unable to bind to 
DNA, since Id proteins lack a basic DNA-binding domain. Four Id genes (Id1-Id4) have been identified to date. These genes localize to various chromosomes and exhibit differences in their pattern of expression and function. The fundamental expression of Id proteins has an important role in the stimulation of cell growth and inhibition of cell differentiation. High expression of Id1 in aggressive BCs suggests that Id1 may act as a key regulator of BC development (50). Further, Id proteins stimulate proliferation, angiogenesis, invasion, and metastasis in BC cells. Recently, Id1 is identified as an oncogene not only since it is upregulated in a wide range of human tumors and relates to poor clinical outcomes and reduced survival but also since it is related to cell growth, and aggressive behavior in many types of human cancer cells. Several studies demonstrated that overexpression of Id 1 is a prognostic factor for aggressive breast tumors (51).

\subsection{Lysine Specific Demethylase 1(LSD1)}

Demethylation is a fundamental epigenetic mechanism in controlling gene transcription, which is related to the suppression of transcription (52). LSD1 is a nuclear amine oxidase that specifically removes monoor di-methyl groups from lysine 4 on histone $\mathrm{H3}$ by Flavin-adenine-dinucleotide(FAD)-dependent oxidative reaction (53). LSD1 interacts with several transcriptional corepressor complexes, including Histone deacetylase 1/2 (HDAC1/2), repressor element-1 silencing transcription factor (REST) corepressor 1 (Co-REST), and C-terminal binding protein (CtBP). LSD1 permits corepressor complexes to selectively initiate or suppress transcription, thereby playing a vital role in the regulation of gene expression (54). The regulation of gene expression by LSD1 has been revealed to be essential in several biological operations such as organogenesis and differentiation. In addition, LSD1 is involved in many signaling pathways that are key for cell growth, survival, and epithelial-mesenchymal transition (EMT) (55).

Epigenetic regulation of gene expression is implicated in multiple tumor types, including BC. LSD1 is overexpressed in BC cells, whereas the importance of this overexpression is not clear.

Vasilatos et al. have indicated that interaction LSD1 and HDACs at chromatin level is dysregulated in BC cells, resulting in aberrant gene expression that can induce breast tumorigenesis. Also, they revealed that LSD1 inhibition prevented the proliferation of $\mathrm{BC}$ cells. According to these studies, LSD1 is considered a tumor-promoting enzyme in BC (56).

Nagasawa et al. indicated that overexpression of LSD1 is a poor prognostic factor in aggressive BCs. They predicted that the expression of LSD1 is related to poor recurrencefree survival in patients with aggressive BC using bioinformatics tools (57). In another study, Lim et al. indicated that
LSD1 might provide a predictive marker for aggressive biology of ER-negative BCs (54).

\subsection{Protein Kinase C (PKC)}

Protein kinase $\mathrm{C}(\mathrm{PKC})$ is a family of serine/threonine kinases, which controls signal transduction pathways involved in cell growth, survival, differentiation, migration, and invasion. The PKC family consists of at least 12 isoforms that are categorized into 3 groups according to the structure of regulatory domains, including classical $(\alpha, \beta \mathrm{I}, \beta \mathrm{II}$, and $\gamma)$, novel $(\delta, \varepsilon, \eta, \mu$, and $\theta)$, and atypical $(\lambda, \iota$, and $\zeta)$. Activation of classical PKCs depends on $\mathrm{Ca}^{2+}$ and diacylglycerol (DAG). Novel PKCs are activated by DAG, and atypical PKCs are activated independently of both $\mathrm{Ca}^{2+}$ and DAG (58). Various PKC isoforms are involved in tumorigenesis in various tissues; therefore, it is vital to determine the special PKC isoform that is important for BC progression.

Some studies have revealed an enhanced expression of PKC activity in malignant BCs. They demonstrated that elevated PKC- $\alpha$ expression is related to the elevated movement and aggressiveness of BC cells. PKC- $\alpha$ overexpression results in an increased rate of proliferation, more effective anchorage-independent growth, substantial changes in cellular morphology characterized through loss of an epithelioid morphology with a marked enhance in the expression of vimentin, a marked reduction in ER mRNA transcripts and estrogen-dependent gene expression, and increased tumorigenesis and metastasis when injected into nude mice (59). A reverse connection between PKC$\alpha$ activity and ER expression was established in BC cells so that ER- BC cells express higher levels of PKC- $\alpha$ than the levels expressed in ER + cancer cells. PKC- $\delta$ has been demonstrated to act as a tumor suppressor and enhances apoptosis in breast carcinoma cells (60). Nevertheless, a direct connection between PKC- $\varepsilon$ and BC has not been established to date. The results of Pan et al.'s study showed that PKC- $\varepsilon$ was critically involved in establishing an aggressive and motile phenotype in $\mathrm{BC}$ (61). The results of several studies indicate that PKC- $\alpha$, PKC- $\delta$, PKC- $\varepsilon$, and PKC $\iota / \lambda$ are unique prognostic factors in aggressive BC. Also, these studies have indicated that overexpression of PKCs is related to the reduction of disease-free survival (62).

\subsection{Transcription Factor AP2}

AP2 is a DNA-binding protein, which exerts both activating and suppressing effects on target genes. The AP2 family is constituted of 3 extremely homologous, retinoic acid (RA)-inducible proteins, including $\mathrm{AP} 2 \alpha, \mathrm{AP} 2 \beta$, and AP2 $\gamma$ (63). AP2 proteins regulate gene expression through binding to consensus DNA sequences within target gene promoters and have a crucial role in cell growth, differentiation, and apoptosis (64). Alterations in the function of AP2 are related to the malignant phenotype. Also, AP2 is recently suggested as a novel tumor suppressor gene. In 
$\mathrm{BC}$, low nuclear expression of AP2 is related to disease development and enhanced metastatic potential of tumor cells. Moreover, AP2 appears to be involved in the control of genes such as HER2 and ER that are altered in BC; therefore, it may play a key role in the prognosis and progression of BC (65). Pellikainen et al. indicated that AP2 played a prognostic role in BC. They also demonstrated that decreased nuclear expression of AP2 independently can predict the enhanced risk of recurrent disease in patients with $\mathrm{BC}(66)$.

\subsection{Urokinase Plasminogen Activator (UPA)}

Urokinase plasminogen activator (UPA) is one of the essential mediators of cancer invasion and metastasis. Indeed, uPA is a serine protease, which converts zymogen plasminogen to active plasmin. Plasmin affects broad types of protein substrates such as constituents of ECM. Also, plasmin mediates the degradation of ECM by activation of certain MMPs that, in turn, increases proteolysis of ECM (67). Plasminogen activator inhibitor 1 (PAI1), an inhibitor of uPA, also plays a major role in tissue remodeling and degradation of ECM. In addition to this activity, uPA and PAI1 have other activities that allow them to play a role in invasion. These roles include induction of angiogenesis, an increase of cell growth, enhancement of cell migration, modulation of cell adhesion, inhibition of apoptosis, and activation of special growth factors (68). Moreover, uPA and PAI1 are overexpressed in several cancer cells such as BC. Different groups have reported that great levels of uPA and PAI1 activity in patients with BC correlate with shorter disease-free survival and a higher risk of primary disease recurrence. In these studies, uPA and PAI1 are considered independent and novel prognostic factors of aggressiveness in patients with BC (69).

\subsection{Activated Leukocyte Cell Adhesion Molecule (ALCAM)}

ALCAM is a glycoprotein of the immunoglobulin superfamily expressed by endothelial, epithelial, neuronal, and hematopoietic cells. This molecule is involved in cellular adhesion and promotes the growth and aggressiveness of tumor cells. ALCAM brings tumor cells together by homotypic interactions, thereby inhibiting interactions with neighbor endothelium that can simplify metastasis (70). King et al. found that levels of ALCAM transcripts are reduced in early tumors of breast carcinoma. They also indicated that reduced expression of ALCAM correlated with poor prognosis and more aggressive phenotype in patients with BC (71). The results of several studies demonstrated that ALCAM expression is a new prognostic factor in $\mathrm{BC}(72)$.

\subsection{Aldehyde Dehydrogenase 1(ALDH1)}

ALDH1, a detoxifying enzyme, is responsible for the oxidation of intracellular aldehydes and, hence, protects stem cells from oxidative stress. In addition, ALDH1 may affect primary differentiation and proliferation of stem cells by converting retinol into retinoic acid, a modulator of cell growth (73). High activity of ALDH1 is characteristic of BC and plays a key role in mediating aggressive behavior of this type of cancer. Therefore, the expression of this stem cell marker is related to the poor prognosis of BC. The prognostic value of ALDH1 in aggressive BC has been reported through different groups (74). Other stem cell markers such as signal transducer CD24 (CD24), cell surface adhesion receptor (CD44), Kruppel-like factor 4 (KLF4), Kruppellike factor 5 (KLF5), and sex-determining region Y (SRY)Box2 (SOX2), as well as ALDH1, have also been identified as new prognostic factors in aggressive BC (75).

Daisuke Yamashita et al. in 2020 introduced a new molecular signature for targeted therapy in BC. These researchers showed that targeting aldehydedehydrogenase1A3 (ALDH1A3) during migration and circulating tumor cell adhesion in metastasis cascade of BC brain metastases (BCBM) could be a new attractive therapeutic target as a metastatic-guiding molecule for the management of BC and other similar types of cancers (76).

\subsection{Forkhead Box Transcription Factor C1 (FOXC1)}

FOXC1 plays key roles in cellular growth, migration, differentiation, survival, and longevity. The different studies showed that overexpression of FOXC1 is related to an aggressive phenotype in BC, as it induces EMT and increases cell growth, migration, and invasion in these cells. They also identified FOXC1 as a new prognostic factor in aggressive forms of BC (77). Some recent prognostic studies on the above-mentioned factors and their relationship with clinical outcomes in patients with aggressive forms of BC are summarized in Table 1.

\section{Conclusions}

We discussed the prognostic value of new factors in patients with aggressive $\mathrm{BC}$ in this review. Conventional prognostic factors in BC, including lymph node status and tumor size, and molecular markers such as PR, ER, HER2, and Ki-67 cannot accurately predict the aggressive behavior of most BCs. Therefore, identification of such factors involved in metastasis and invasion of $\mathrm{BC}$ is of great importance in the development of therapeutic targets to combat BC.

\section{Acknowledgments}

The authors express their sincere gratitude to the pharmaceutical sciences research center of Ardabil University of Medical Sciences for their sincere cooperation. 


\begin{tabular}{|c|c|c|c|c|}
\hline Prognostic Factor & Patients & Assay & DFS/OS & Study \\
\hline P53 & 217 & IHC & ND & Guerra et al. (24) \\
\hline P53 & 126 & SSCP & Reduced & Shao et al. (78) \\
\hline MMP2 & 453 & IHC & Shortened & Talvensaari-Mattila et al. (79) \\
\hline MMP9 & 113 & Gelatin quantitative zymography & Shortened & Ranuncolo et al. (80) \\
\hline MMP13 & 263 & IHC & Decreased & Zhang et al. (81) \\
\hline MMP14 & 120 & IHC & Shortened & Jiang et al. (82) \\
\hline MMP9 & 303 & ELISA+ IHC & Reduced & Wang et al. (30) \\
\hline MMP1 & 179 & IHC & Worse & Wang et al. (83) \\
\hline EZH2 & 432 & IHC & Shortened & Jang et al. (32) \\
\hline Fascin & 210 & IHC & Decreased & Yoder et al. (37) \\
\hline HIF1 $\alpha$ & 206 & IHC & Shortened & Schindl et al. (84) \\
\hline HIF2 $\alpha$ & 124 & IHC & Reduced & Wang et al. (44) \\
\hline hTERT & 611 & IHC & Reduced & Poremba et al. (48) \\
\hline hTERT & 121 & RT-PCR & ND & Divella et al. (85) \\
\hline Id1 & 191 & IHC & Shortened & Schoppmann et al. (49) \\
\hline Id1 & 58 & IHC & ND & Fong et al. (51) \\
\hline LSD1 & 32 & IHC & Shortened & Nagasawa et al. (57) \\
\hline LSD1 & 460 & IHC & Shortened & Derr et al. (86) \\
\hline PKC- $\varepsilon$ & 160 & IHC & Reduced & Pan et al. (61) \\
\hline PKC- $\alpha$ & 107 & IHC & Worse & Hsu et al. (62) \\
\hline AP2 & 520 & IHC & Reduced & Pellikainen et al. (66) \\
\hline PAI1 & 73 & ELISA & Shortened & Buta et al. (68) \\
\hline UPA & 166 & IHC & Shortened & Duffy et al. (87) \\
\hline uPA/PAI1 & 606 & ELISA & ND & Lampelj et al. (69) \\
\hline ALCAM & 120 & IHC & Reduced & King et al. (71) \\
\hline ALCAM & 157 & ELISA & Shortened & Witzel et al. (72) \\
\hline ALDH1 & 574 & IHC & Reduced & Mieog et al. (73) \\
\hline ALDH1 & 158 & IHC & Shortened & Ma et al. (74) \\
\hline CD24 & 104 & IHC & Shortened & Surowiak et al. (88) \\
\hline KLF4 & 146 & IHC & Reduced & Pandya et al. (89) \\
\hline KLF5 & 90 & RT-PCR & Shortened & Tong et al. (90) \\
\hline SOX2 & 117 & IHC & Shortened & Finicelli et al. (91) \\
\hline FOXC1 & 253 & IHC & Reduced & Xu et al. (77) \\
\hline FOXC1 & 118 & IHC & Reduced & Jensen et al. (92) \\
\hline
\end{tabular}

Abbreviations: IHC, immunohistochemistry; SSCP, single-strand conformation polymorphism analysis; ELISA, enzyme-linked immunosorbent; RT-PCR, real-time polymerase chain reaction; $\mathrm{ND}$, not done.

\section{Footnotes}

Authors' Contribution: K.N. contributed to the design and writing of the paper. S.F.A and Y.P. contributed to data collection, writing the paper, and providing the Tables. A. A. contributed to the design of the paper and revised the final version of the paper.

Conflict of Interests: The authors declare that they have no known competing financial interests or personal relationships.

Funding/Support: Ardabil University of Medical Sciences

\section{References}

1. Nejati-Koshki K, Akbarzadeh A, Pourhassan-Moghaddam M. Curcumin inhibits leptin gene expression and secretion in breast cancer cells by estrogen receptors. Cancer Cell Int. 2014;14:66. doi: 10.1186/1475-2867-14-66. [PubMed: 25866478]. [PubMed Central: PMC4392783].

2. Panahi Y, Mohammadhosseini M, Nejati-Koshki K, Abadi AJ, Moafi HF, Akbarzadeh A, et al. Preparation, surface properties, and therapeutic applications of gold nanoparticles in biomedicine. Drug Res. 2017;67(2):77-87. doi: 10.1055/s-0042-115171. [PubMed: 27824433].

3. Pourhassan-Moghaddam M, Zarghami N, Mohsenifar A, RahmatiYamchi M, Gholizadeh D, Akbarzadeh A, et al. Watercress-based gold nanoparticles: biosynthesis, mechanism of formation and study of their biocompatibility in vitro. Micro Nano Lett. 2014;9(5):345-50. doi: 10.1049/mnl.2014.0063. 
4. Nejati K, Dadashpour M, Gharibi T, Mellatyar H, Akbarzadeh A. Biomedical applications of functionalized gold nanoparticles: A review. J Clust Sci. 2021:1-16. doi: 10.1007/s10876-020-01955-9.

5. Ellis M, Hayes D, Lippman M. Diseases of the breast. Philadelpha, USA: Lippincott-Raven; 2000.

6. Castillo-Guardiola V, Sarabia-Meseguer MD, Marin-Vera M, SanchezBermudez AI, Alonso-Romero JL, Noguera-Velasco JA, et al. New insights into the performance of multigene panel testing: Two novel nonsense variants in BRIP1 and TP53 in a young woman with breast cancer. Cancer Genet. 2018;228-229:1-4. doi: 10.1016/j.cancergen.2018.06.002. [PubMed: 30553462].

7. Dixon K, Kopras E. Genetic alterations and DNA repair in human carcinogenesis. Semin Cancer Biol. 2004;14(6):441-8. doi: 10.1016/j.semcancer.2004.06.007. [PubMed:15489137].

8. Welcsh PL, Owens KN, King MC. Insights into the functions of BRCA1 and BRCA2. Trends Genet. 2000;16(2):69-74. doi: 10.1016/s01689525(99)01930-7. [PubMed:10652533].

9. Bianchini G, Balko JM, Mayer IA, Sanders ME, Gianni L. Triple-negative breast cancer: Challenges and opportunities of a heterogeneous disease. Nat Rev Clin Oncol. 2016;13(11):674-90. doi: 10.1038/nrclinonc.2016.66. [PubMed: 27184417]. [PubMed Central: PMC5461122].

10. Narod SA, Foulkes WD. BRCA1 and BRCA2: 1994 and beyond. Nat Rev Cancer. 2004;4(9):665-76. doi: 10.1038/nrc1431. [PubMed: 15343273]

11. Pinto PMT. Phenotypic and genotypic heterogeneity of hereditary breas and ovarian cancer [dissertation]. Porto, Portugal: University of Porto; 2017.

12. Pahlavan Y, Mohammadi Nasr M, Dalir Abdolahinia E, Pirdel Z, Razi Soofiyani S, Siahpoush S, et al. Prominent roles of microRNA-142 in cancer. Pathol Res Pract. 2020;216(11):153220. doi: 10.1016/j.prp.2020.153220. [PubMed: 33007646].

13. Nejati K, Alivand M, Arabzadeh A. MicroRNA-22 in female malignancies: Focusing on breast, cervical, and ovarian cancers. Pathol Res Pract. 2021;223:153452. doi: 10.1016/j.prp.2021.153452. [PubMed: 33993061].

14. Bahmanpour Z, Sheervalilou R, Choupani J, Shekari Khaniani M, Montazeri V, Mansoori Derakhshan S. A new insight on serum microRNA expression as novel biomarkers in breast cancer patients. JCell Physiol. 2019;234(11):19199-211. doi: 10.1002/jcp.28656. [PubMed: 31026062]

15. Chambers AF, Naumov GN, Varghese HJ, Nadkarni KV, MacDonald IC, Groom AC. Critical steps in hematogenous metastasis: An overview. Surg Oncol Clin N Am. 2001;10(2):243-55. vii. [PubMed: 11382585].

16. Barroso-Sousa R, Keenan TE, Pernas S, Exman P, Jain E, GarridoCastro AC, et al. Tumor mutational burden and PTEN alterations as molecular correlates of response to PD-1/L1 blockade in metastatic triple-negative breast cancer. Clin Cancer Res. 2020;26(11):2565-72. doi: 10.1158/1078-0432.CCR-19-3507. [PubMed: 32019858]. [PubMed Central: PMC7269810]

17. Lehmann BD, Abramson VG, Sanders ME, Mayer EL, Haddad TC, Nanda R, et al. TBCRC 032 IB/II multicenter study: Molecular insights to ar antagonist and PI3K inhibitor efficacy in patients with $\mathrm{AR}(+)$ metastatic triple-negative breast cancer. Clin Cancer Res. 2020;26(9):2111-23. doi: 10.1158/1078-0432.CCR-19-2170. [PubMed: 31822498]. [PubMed Central: PMC7196503].

18. Hayes DF, Trock B, Harris AL. Assessing the clinical impact of prognostic factors: When is "statistically significant" clinically useful? Breast Cancer Res Treat. 1998;52(1-3):305-19. doi: 10.1023/a:1006197805041. [PubMed: 10066089].

19. Gharibi T, Babaloo Z, Hosseini A, Abdollahpour-Alitappeh M, Hashemi $\mathrm{V}$, Marofi $\mathrm{F}$, et al. Targeting STAT3 in cancer and autoimmune diseases. Eur J Pharmacol. 2020;878:173107. doi: 10.1016/j.ejphar.2020.173107. [PubMed: 32278856].

20. Lacroix M, Toillon RA, Leclercq G. p53 and breast cancer, an update. Endocr Relat Cancer. 2006;13(2):293-325. doi:10.1677/erc.1.01172. [PubMed: 16728565].

21. Zohre S, Kazem NK, Abolfazl A, Mohammad RY, Aliakbar M, Effat A, et al. Trichostatin A-induced apoptosis is mediated by Kruppellike factor 4 in ovarian and lung cancer. Asian Pac J Cancer Prev. 2014;15(16):6581-6. doi: 10.7314/apjcp.2014.15.16.6581. [PubMed: 25169491].
22. Sheikholeslami K, Ali Sher A, Lockman S, Kroft D, Ganjibakhsh M Nejati-Koshki K, et al. Simvastatin induces apoptosis in medulloblastoma brain tumor cells via mevalonate cascade prenylation substrates. Cancers. 2019;11(7). doi: 10.3390/cancers11070994. [PubMed: 31319483]. [PubMed Central: PMC6678292].

23. Mackay J, Steel CM, Elder PA, Forrest AP, Evans HJ. Allele loss on short arm of chromosome 17 in breast cancers. Lancet. 1988;2(8625):1384-5. doi: 10.1016/s0140-6736(88)90584-3. [PubMed: 2904522].

24. Guerra E, Cimadamore A, Simeone P, Vacca G, Lattanzio R, Botti G, et al. p53, cathepsin D, Bcl-2 are joint prognostic indicators of breast cancer metastatic spreading. BMC Cancer. 2016;16:649. doi:10.1186/s12885016-2713-3. [PubMed: 27538498]. [PubMed Central: PMC4991058].

25. Abdelmaksoud-Dammak R, Saadallah-Kallel A, Miladi-Abdennadher I, Ayedi L, Khabir A, Sallemi-Boudawara T, et al. CpG methylation of ubiquitin carboxyl-terminal hydrolase 1 (UCHL1) and P53 mutation pattern in sporadic colorectal cancer. Tumour Biol. 2016;37(2):1707-14. doi: 10.1007/s13277-015-3902-4. [PubMed: 26314856].

26. Liu S, Gonzalez-Prieto R, Zhang M, Geurink PP, Kooij R, Iyengar PV, et al. Deubiquitinase activity profiling identifies UCHL1 as a candidate oncoprotein that promotes TGFbeta-induced breast cancer metastasis. Clin Cancer Res. 2020;26(6):1460-73. doi: 10.1158/1078-0432.CCR-191373. [PubMed: 31857432]. [PubMed Central: PMC7611208].

27. Singer CF, Kronsteiner N, Marton E, Kubista M, Cullen KJ, Hirtenlehner $\mathrm{K}$, et al. MMP-2 and MMP-9 expression in breast cancerderived human fibroblasts is differentially regulated by stromalepithelial interactions. Breast Cancer Res Treat. 2002;72(1):69-77. doi: 10.1023/a:1014918512569. [PubMed: 12000221].

28. Egeblad M, Werb Z. New functions for the matrix metalloproteinases in cancer progression. Nat Rev Cancer. 2002;2(3):161-74. doi: 10.1038/nrc745. [PubMed: 11990853].

29. Iwata $\mathrm{H}$, Kobayashi S, Iwase H, Masaoka A, Fujimoto N, Okada Y Production of matrix metalloproteinases and tissue inhibitors of metalloproteinases in human breast carcinomas. Jpn J Cancer Res. 1996;87(6):602-11. doi: 10.1111/j.1349-7006.1996.tb00266.x. [PubMed 8766524]. [PubMed Central: PMC5921148].

30. Wang RX, Chen S, Huang L, Shao ZM. Predictive and prognostic value of Matrix metalloproteinase (MMP) - 9 in neoadjuvant chemotherapy for triple-negative breast cancer patients. BMC Cancer. 2018;18(1):909. doi: 10.1186/s12885-018-4822-7. [PubMed: 30241470]. [PubMed Central: PMC6151029].

31. Doherty LF, Bromer JG, Zhou Y, Aldad TS, Taylor HS. In utero exposure to diethylstilbestrol (DES) or bisphenol-A (BPA) increases EZH2 expression in the mammary gland: an epigenetic mechanism linking endocrine disruptors to breast cancer. Horm Cancer. 2010;1(3):146-55. doi: 10.1007/s12672-010-0015-9. [PubMed: 21761357]. [PubMed Central: PMC3140020].

32. Jang SH, Lee JE, Oh MH, Lee JH, Cho HD, Kim KJ, et al. High EZH2 protein expression is associated with poor overall survival in patients with luminal a breast cancer. J Breast Cancer. 2016;19(1):53-60. doi: 10.4048/jbc.2016.19.1.53. [PubMed: 27066096]. [PubMed Central: PMC4822107].

33. Collett K, Eide GE, Arnes J, Stefansson IM, Eide J, Braaten A, et al. Expression of enhancer of zeste homologue 2 is significantly associated with increased tumor cell proliferation and is a marker of aggressive breast cancer. Clin Cancer Res. 2006;12(4):1168-74. doi:10.1158/10780432.CCR-05-1533. [PubMed: 16489070]

34. Snyder M, Huang XY, Zhang JJ. Signal transducers and activators of transcription 3 (STAT3) directly regulates cytokine-induced fascin expression and is required for breast cancer cell migration. J Biol Chem. 2011;286(45):38886-93. doi: 10.1074/jbc.M111.286245. [PubMed: 21937440]. [PubMed Central: PMC3234713].

35. Sun J, He H, Pillai S, Xiong Y, Challa S, Xu L, et al. GATA3 transcription factor abrogates Smad4 transcription factor-mediated fascin overexpression, invadopodium formation, and breast cancer cell invasion. J Biol Chem. 2013;288(52):36971-82. doi: 10.1074/jbc.M113.506535. [PubMed: 24235142]. [PubMed Central: PMC3873555].

36. Ghebeh H, Al-Khaldi S, Olabi S, Al-Dhfyan A, Al-Mohanna F, Barnawi $\mathrm{R}$, et al. Fascin is involved in the chemotherapeutic resistance of breast cancer cells predominantly via the PI3K/Akt pathway. BrJ Can- 
cer. 2014;111(8):1552-61. doi: 10.1038/bjc.2014.453. [PubMed: 25117814] [PubMed Central: PMC4200093].

37. Yoder BJ, Tso E, Skacel M, Pettay J, Tarr S, Budd T, et al. The expression of fascin, an actin-bundling motility protein, correlates with hormone receptor-negative breast cancer and a more aggressive clinical course. Clin Cancer Res. 2005;11(1):186-92. [PubMed:15671545].

38. Grothey A, Hashizume R, Tubb BE, Patrick CW, Mooney EE, McCrea PD. C-erbB-2/ HER-2 upregulates fascin, an actin-bundling protein associated with cell motility, in human breast cancer cell lines. Oncogene. 2000;19(42):4864-75. doi: 10.1038/sj.onc.1203838. [PubMed 11039904].

39. De Francesco EM, Lappano R, Santolla MF, Marsico S, Caruso A, Maggiolini M. HIF-1alpha/GPER signaling mediates the expression of VEGF induced by hypoxia in breast cancer associated fibroblasts (CAFs). Breast Cancer Res. 2013;15(4):R64. doi: 10.1186/bcr3458. [PubMed: 23947803]. [PubMed Central: PMC3978922].

40. Blancher C, Moore JW, Talks KL, Houlbrook S, Harris AL. Relationship of hypoxia-inducible factor(HIF)-1alpha and HIF-2alpha expression to vascular endothelial growth factor induction and hypoxia survival in human breast cancer cell lines. Cancer Res. 2000;60(24):7106-13. [PubMed: 11156418].

41. Choi JY, Jang YS, Min SY, Song JY. Overexpression of MMP-9 and HIF1alpha in breast cancer cells under hypoxic conditions. J Breast Cancer. 2011;14(2):88-95. doi: 10.4048/jbc.2011.14.2.88. [PubMed: 21847402] [PubMed Central: PMC3148536].

42. Kaluz S, Kaluzova M, Stanbridge EJ. Regulation of gene expression by hypoxia: Integration of the HIF-transduced hypoxic signal at the hypoxia-responsive element. Clin Chim Acta. 2008;395(1-2):6-13. doi: 10.1016/j.cca.2008.05.002. [PubMed: 18505681]. [PubMed Central: PMC2536516].

43. Ryan HE, Poloni M, McNulty W, Elson D, Gassmann M, Arbeit JM, et al. Hypoxia-inducible factor-1alpha is a positive factor in solid tumor growth. Cancer Res. 2000;60(15):4010-5. [PubMed: 10945599].

44. Wang HX, Qin C, Han FY, Wang XH, Li N. HIF-2alpha as a prognostic marker for breast cancer progression and patient survival. Genet Mol Res. 2014;13(2):2817-26. doi: 10.4238/2014.January.22.6. [PubMed: 24535905].

45. Fekri Aval S, Akbarzadeh A, Yamchi MR, Zarghami F, Nejati-Koshki K, Zarghami N. Gene silencing effect of SiRNA-magnetic modified with biodegradable copolymer nanoparticles on hTERT gene expression in lung cancer cell line. Artif Cells Nanomed Biotechnol. 2016;44(1):188-93. doi: 10.3109/21691401.2014.934456. [PubMed: 25020049].

46. Kirkpatrick KL, Ogunkolade W, Elkak AE, Bustin S, Jenkins P, Ghilchick M, et al. hTERT expression in human breast cancer and non-cancerous breast tissue: Correlation with tumour stage and c-Myc expression. Breast Cancer Res Treat. 2003;77(3):277-84. doi: 10.1023/a:1021849217054. [PubMed: 12602927].

47. Ceja-Rangel HA, Sanchez-Suarez P, Castellanos-Juarez E, PenarojaFlores R, Arenas-Aranda DJ, Gariglio P, et al. Shorter telomeres and high telomerase activity correlate with a highly aggressive phenotype in breast cancer cell lines. Tumour Biol. 2016;37(9):11917-26. doi 10.1007/s13277-016-5045-7. [PubMed: 27072825].

48. Poremba C, Heine B, Diallo R, Heinecke A, Wai D, Schaefer KL, et al. Telomerase as a prognostic marker in breast cancer: highthroughput tissue microarray analysis of hTERT and hTR. J Pathol. 2002;198(2):181-9. doi: 10.1002/path.1191. [PubMed: 12237877].

49. Schoppmann SF, Schindl M, Bayer G, Aumayr K, Dienes J, Horvat R, et al. Overexpression of Id- 1 is associated with poor clinical outcome in node negative breast cancer. Int J Cancer. 2003;104(6):677-82. doi: 10.1002/ijc.11009. [PubMed: 12640673].

50. Singh J, Murata K, Itahana Y, Desprez PY. Constitutive expression of the Id-1 promoter in human metastatic breast cancer cells is linked with the loss of NF-1/Rb/HDAC-1 transcription repressor complex. Oncogene. 2002;21(12):1812-22. doi: 10.1038/sj.onc.1205252. [PubMed 11896613].

51. Fong S, Itahana Y, Sumida T, Singh J, Coppe JP, Liu Y, et al. Id1 as a molecular target in therapy for breast cancer cell invasion and metastasis. Proc Natl Acad Sci U S A. 2003;100(23):13543-8. doi: 10.1073/pnas.2230238100. [PubMed: 14578451]. [PubMed Central:
PMC263850].

52. Fattahi M, Sheervalilou R, Hoseinpour N, Valiloo M, Pedram N, Montazeri $\mathrm{V}$, et al. The correlation between Twist 1 and 2 promoter methylation status and clinicopathologic characteristics of patients with breast cancer. Gene Rep. 2020;20:100741. doi: 10.1016/j.genrep.2020.100741.

53. Wu Y, Wang Y, Yang XH, Kang $\mathrm{T}$, Zhao Y, Wang $\mathrm{C}$, et al. The deubiquitinase USP28 stabilizes LSD1 and confers stem-celllike traits to breast cancer cells. Cell Rep. 2013;5(1):224-36. doi: 10.1016/j.celrep.2013.08.030. [PubMed: 24075993]. [PubMed Central: PMC4004762].

54. Lim S, Janzer A, Becker A, Zimmer A, Schule R, Buettner R, et al. Lysine-specific demethylase (LSD1) is highly expressed in ER-negative breast cancers and a biomarker predicting aggressive biology. Carcinogenesis. 2010;31(3):512-20. doi: 10.1093/carcin/bgp324. [PubMed: 20042638].

55. Wu ZQ, Li XY, Hu CY, Ford M, Kleer CG, Weiss SJ. Canonical Wnt signaling regulates Slug activity and links epithelial-mesenchymal transition with epigenetic Breast Cancer 1, Early Onset (BRCA1) repression. Proc Natl Acad Sci U S A. 2012;109(41):16654-9. doi: 10.1073/pnas.1205822109. [PubMed: 23011797]. [PubMed Central: PMC3478591].

56. Vasilatos SN, Katz TA, Oesterreich S, Wan Y, Davidson NE, Huang Y. Crosstalk between lysine-specific demethylase 1 (LSD1) and histone deacetylases mediates antineoplastic efficacy of HDAC inhibitors in human breast cancer cells. Carcinogenesis. 2013;34(6):1196-207. doi: 10.1093/carcin/bgt033. [PubMed: 23354309]. [PubMed Central: PMC3670252].

57. Nagasawa S, Sedukhina AS, Nakagawa Y, Maeda I, Kubota M, Ohnuma $S$, et al. LSD1 overexpression is associated with poor prognosis in basal-like breast cancer, and sensitivity to PARP inhibition. PLoS One.2015;10(2). e0118002. doi: 10.1371/journal.pone.0118002.[PubMed: 25679396]. [PubMed Central: PMC4332491].

58. Koivunen J, Aaltonen V, Peltonen J. Protein kinase C (PKC) family in cancer progression. Cancer Lett. 2006;235(1):1-10. doi: 10.1016/j.canlet.2005.03.033. [PubMed:15907369].

59. Ways DK, Kukoly CA, deVente J, Hooker JL, Bryant WO, Posekany $\mathrm{KJ}$, et al. MCF-7 breast cancer cells transfected with protein kinase $\mathrm{C}$-alpha exhibit altered expression of other protein kinase $\mathrm{C}$ isoforms and display a more aggressive neoplastic phenotype. J Clin Invest. 1995;95(4):1906-15. doi: 10.1172/JCI117872. [PubMed: 7706498]. [PubMed Central: PMC295735].

60. Sledge GW, Gokmen-Polar Y. Protein kinase C-beta as a therapeutic target in breast cancer. Semin Oncol. 2006;33(3 Suppl 9):S15-8. doi: 10.1053/j.seminoncol.2006.03.019. [PubMed:16797377].

61. Pan Q, Bao LW, Kleer CG, Sabel MS, Griffith KA, Teknos TN, et al. Protein kinase $C$ epsilon is a predictive biomarker of aggressive breast cancer and a validated target for RNA interference anticancer therapy. Cancer Res. 2005;65(18):8366-71. doi: 10.1158/0008-5472.CAN-05-0553. [PubMed: 16166314].

62. Hsu YH, Yao J, Chan LC, Wu TJ, Hsu JL, Fang YF, et al. Definition of PKCalpha, CDK6, and MET as therapeutic targets in triple-negative breast cancer. Cancer Res. 2014;74(17):4822-35. doi: 10.1158/0008-5472.CAN-140584. [PubMed: 24970481]. [PubMed Central: PMC4154991].

63. Wankhade S, Yu Y, Weinberg J, Tainsky MA, Kannan P. Characterization of the activation domains of AP-2 family transcription factors. J Biol Chem. 2000;275(38):29701-8. doi: 10.1074/jbc.M000931200. [PubMed: 10899156].

64. Hilger-Eversheim K, Moser M, Schorle H, Buettner R. Regulatory roles of AP-2 transcription factors in vertebrate development, apoptosis and cell-cycle control. Gene. 2000;260(1-2):1-12. doi: 10.1016/s03781119(00)00454-6. [PubMed: 11137286].

65. Bosher JM, Williams T, Hurst HC. The developmentally regulated transcription factor AP-2 is involved in c-erbB-2 overexpression in human mammary carcinoma. Proc Natl Acad Sci U S A. 1995;92(3):7447. doi: 10.1073/pnas.92.3.744. [PubMed: 7846046]. [PubMed Central: PMC42696].

66. Pellikainen J, Kataja V, Ropponen K, Kellokoski J, Pietilainen T, Bohm J, et al. Reduced nuclear expression of transcription factor AP-2 asso- 
ciates with aggressive breast cancer. Clin Cancer Res. 2002;8(11):348795. [PubMed: 12429639].

67. Duffy MJ. Urokinase plasminogen activator and its inhibitor, PAI-1, as prognostic markers in breast cancer: from pilot to level 1 evidence studies. Clin Chem. 2002;48(8):1194-7. [PubMed: 12142372].

68. Buta M, Dzodic R, Durisic I, Markovic I, Vujasinovic T, Markicevic $\mathrm{M}$, et al. Potential clinical relevance of UPA and PAI-1 levels in nodenegative, postmenopausal breast cancer patients bearing histological grade II tumors with ER/PR expression, during an early followup. Tumour Biol. 2015;36(10):8193-200. doi: 10.1007/s13277-015-3573-1. [PubMed: 25994573].

69. Lampelj M, Arko D, Cas-Sikosek N, Kavalar R, Ravnik M, JezersekNovakovic B, et al. Urokinase plasminogen activator (uPA) and plasminogen activator inhibitor type-1 (PAI-1) in breast cancer - correlation with traditional prognostic factors. Radiol Oncol. 2015;49(4):35764. doi: 10.2478/raon-2014-0049. [PubMed: 26834522]. [PubMed Central: PMC4722926].

70. Ofori-Acquah SF, King JA. Activated leukocyte cell adhesion molecule: A new paradox in cancer. Transl Res. 2008;151(3):122-8. doi: 10.1016/j.trsl.2007.09.006. [PubMed: 18279810].

71. King JA, Ofori-Acquah SF, Stevens T, Al-Mehdi AB, Fodstad O, Jiang WG. Activated leukocyte cell adhesion molecule in breast cancer: Prognostic indicator. Breast Cancer Res. 2004;6(5):R478-87. doi: 10.1186/bcr815. [PubMed: 15318930]. [PubMed Central: PMC549164].

72. Witzel I, Schroder C, Muller V, Zander H, Tachezy M, Ihnen M, et al. Detection of activated leukocyte cell adhesion molecule in the serum of breast cancer patients and implications for prognosis. Oncology. 2012;82(6):305-12. doi: 10.1159/000337222. [PubMed: 22555284].

73. Mieog JS, de Kruijf EM, Bastiaannet E, Kuppen PJ, Sajet A, de Craen AJ, et al. Age determines the prognostic role of the cancer stem cell marker aldehyde dehydrogenase-1 in breast cancer. BMC Cancer. 2012;12:42. doi: 10.1186/1471-2407-12-42. [PubMed: 22280212]. [PubMed Central: PMC3292491].

74. Ma F, Li H, Li Y, Ding X, Wang H, Fan Y, et al. Aldehyde dehydrogenase 1 (ALDH1) expression is an independent prognostic factor in triple negative breast cancer (TNBC). Medicine. 2017;96(14). e6561. doi: 10.1097/MD.0000000000006561. [PubMed: 28383433]. [PubMed Central: PMC5411217].

75. O'Conor CJ, Chen T, Gonzalez I, Cao D, Peng Y. Cancer stem cells in triple-negative breast cancer: A potential target and prognostic marker. Biomark Med. 2018;12(7):813-20. doi: 10.2217/bmm-2017-0398. [PubMed: 29902924].

76. Yamashita D, Minata M, Ibrahim AN, Yamaguchi S, Coviello V, Bernstock JD, et al. Identification of ALDH1A3 as a viable therapeutic target in breast cancer metastasis-initiating cells. Mol Cancer Ther. 2020;19(5):1134-47. doi: 10.1158/1535-7163.MCT-19-0461. [PubMed: 32127468]. [PubMed Central: PMC7716183].

77. Xu YL, Yao R, Li J, Zhou YD, Mao F, Pan B, et al. FOXC1 overexpression is a marker of poor response to anthracycline-based adjuvant chemotherapy in sporadic triple-negative breast cancer. Cancer Chemother Pharmacol. 2017;79(6):1205-13. doi: 10.1007/s00280-0173319-4. [PubMed: 28493031]. [PubMed Central: PMC5438824].

78. Shao ZM, Wu J, Shen ZZ, Nguyen M. p53 mutation in plasma DNA and its prognostic value in breast cancer patients. Clin Cancer Res. 2001;7(8):2222-7. [PubMed: 11489795].

79. Talvensaari-Mattila A, Paakko P, Hoyhtya M, Blanco-Sequeiros G, Turpeenniemi-Hujanen T. Matrix metalloproteinase-2 immunoreactive protein: A marker of aggressiveness in breast carcinoma. Cancer. 1998;83(6):1153-62. doi: 10.1002/(sici)1097- 0142(19980915)83:6<1153::aid-cncr14>3.0.co;2-4. [PubMed: 9740080].

80. Ranuncolo SM, Armanasco E, Cresta C, Bal De Kier Joffe E, Puricelli L Plasma MMP-9 (92 kDa-MMP) activity is useful in the follow-up and in the assessment of prognosis in breast cancer patients. Int J Cancer. 2003;106(5):745-51. doi:10.1002/ijc.11288. [PubMed: 12866035].

81. Zhang B, Cao X, Liu Y, Cao W, Zhang F, Zhang S, et al. Tumor-derived matrix metalloproteinase-13 (MMP-13) correlates with poor prognoses of invasive breast cancer. BMC Cancer. 2008;8:83. doi: 10.1186/1471-2407-8 83. [PubMed: 18373849]. [PubMed Central: PMC2362123].

82. Jiang WG, Davies G, Martin TA, Parr C, Watkins G, Mason MD, et al. Expression of membrane type-1 matrix metalloproteinase, MT1-MMP in human breast cancer and its impact on invasiveness of breast cancer cells. Int J Mol Med. 2006;17(4):583-90. [PubMed: 16525713].

83. Wang J, Ye C, Lu D, Chen Y, Jia Y, Ying X, et al. Matrix metalloproteinase1 expression in breast carcinoma: A marker for unfavorable prog nosis. Oncotarget. 2017;8(53):91379-90. doi:10.18632/oncotarget.20557. [PubMed: 29207651]. [PubMed Central: PMC5710931].

84. Schindl M, Schoppmann SF, Samonigg H, Hausmaninger H, Kwasny $\mathrm{W}$, Gnant M, et al. Overexpression of hypoxia-inducible factor 1alpha is associated with an unfavorable prognosis in lymph node-positive breast cancer. Clin Cancer Res. 2002;8(6):1831-7. [PubMed: 12060624].

85. Divella R, Tommasi S, Lacalamita R, Daniele A, Abbate I, Garrisi VM, et al. Circulating hTERT DNA in early breast cancer. Anticancer Res. 2009;29(7):2845-9. [PubMed: 19596972].

86. Derr RS, van Hoesel AQ, Benard A, Goossens-Beumer IJ, Sajet A, DekkerEnsink NG, et al. High nuclear expression levels of histone-modifying enzymes LSD1, HDAC2 and SIRT1 in tumor cells correlate with decreased survival and increased relapse in breast cancer patients. $B M C$ Cancer. 2014;14:604. doi:10.1186/1471-2407-14-604. [PubMed: 25139823]. [PubMed Central: PMC4148956].

87. Duffy MI, Reilly D, O'Sullivan C, O'Higgins N, Fennelly JJ, Andreasen P. Urokinase-plasminogen activator, a new and independent prognostic marker in breast cancer. Cancer Res. 1990;50(21):6827-9. [PubMed: 2119883].

88. Surowiak P, Materna V, Gyorffy B, Matkowski R, Wojnar A, Maciejczyk A, et al. Multivariate analysis of oestrogen receptor alpha, pS2, metallothionein and CD24 expression in invasive breast cancers. BrJCancer. 2006;95(3):339-46. doi: 10.1038/sj.bjc.6603254. [PubMed: 16892043]. [PubMed Central: PMC2360643].

89. Pandya AY, Talley LI, Frost AR, Fitzgerald TJ, Trivedi V, Chakravarthy $\mathrm{M}$, et al. Nuclear localization of KLF4 is associated with an aggressive phenotype in early-stage breast cancer. Clin Cancer Res. 2004;10(8):2709-19. doi: 10.1158/1078-0432.ccr-03-0484. [PubMed: 15102675].

90. Tong D, Czerwenka K, Heinze G, Ryffel M, Schuster E, Witt A, et al. Expression of KLF5 is a prognostic factor for disease-free survival and overall survival in patients with breast cancer. Clin Cancer Res. 2006;12(8):2442-8. doi: 10.1158/1078-0432.CCR-05-0964. [PubMed: 16638850].

91. Finicelli M, Benedetti G, Squillaro T, Pistilli B, Marcellusi A, Mariani P et al. Expression of stemness genes in primary breast cancer tissues: the role of SOX2 as a prognostic marker for detection of early recurrence. Oncotarget. 2014;5(20):9678-88. doi: 10.18632/oncotarget.1936. [PubMed: 25127259]. [PubMed Central: PMC4259429].

92. Jensen TW, Ray T, Wang J, Li X, Naritoku WY, Han B, et al. Diagnosis of basal-like breast cancer using a FOXC1-based assay. J Natl Cancer Inst. 2015;107(8). doi: 10.1093/jnci/djv148. [PubMed: 26041837]. [PubMed Central: PMC4554196] 